Textures and Microstructures, 1988, Vol. 10, pp. 77-96

Reprints available directly from the publisher

Photocopying permitted by license only

(C) 1988 Gordon and Breach Science Publishers Inc.

Printed in the United Kingdom

\title{
On the Normal Distribution in the Orientation Space
}

\section{S. MATTHIES}

Zentralinstitut für Kernforschung Rossendorf, 8051, Dresden, DDR, PF 19

\section{J. MULLER}

Institut de Mathématiques, 7 rue René Descartes, 67000 Strasbourg, France

G. W. VINEL

Zentralinstitut für Kernforschung Rossendorf, 8051 Dresden, DDR, PF 19

(Received March 15, 1988)

The properties of model distributions used in texture analysis up to now are discussed. The normal distribution in the $G$-space (recently investigated by T. I. Savjolova) is analysed. Its connection with the central limit theorem of probability theory is demonstrated in a mathematically simplified manner. An analytically closed approximative expression (with very high precision for halfwidths of practical interest) for the normal distribution is derived. Possible correlations between forms of texture components and mechanisms of texture development are mentioned.

KEY WORDS: ODF analysis, model functions, normal distribution, component fit.

\section{INTRODUCTION}

The orientation distribution of the crystallites in a polycrystalline sample is of main interest in texture analysis. This distribution is described by the so-called orientation distribution function (ODF) $f(g)$, with $g-a$ set of three numbers unambiguously determining the orientation of a crystal fixed, right-handed cartesian coordinate system $K_{B}$ in regard of a sample fixed coordinate system $K_{A}$ (Bunge, 1982).

Commonly three Eulerian angles (e.g. $\alpha, \beta, \gamma$ ) are used for $g$ 
characterizing three rotations of certain order and manner with the help of which $K_{A}$ can be oriented parallel to $K_{B}$. All possible orientations lie in the " $G$-space"

$$
g=\{\alpha, \beta, \gamma\} ; \quad g \in G: 0 \leqslant \alpha, \gamma<2 \pi, \quad 0 \leqslant \beta \leqslant \pi,
$$

with

$$
d g=d \alpha \sin \beta d \beta d \gamma, \quad \int_{G} d g=8 \pi^{2} .
$$

Another possibility to characterize $g$ is $g=[\omega, \vartheta, \varphi]$. At this $K_{A}$ will be oriented parallel to $K_{B}$ by a rotation around an axis with the direction $\vec{n}\left(\vartheta, \varphi\right.$-its spherical angles in regard of $\left.K_{A}\right)$ through the angle $\omega$ :

$g=[\omega, \vartheta, \varphi] \equiv[\omega, \vec{n}] ; \quad g \in G: 0 \leqslant \varphi<2 \pi, \quad 0 \leqslant \vartheta, \omega \leqslant \pi$,

with

$$
d g=4 \sin ^{2} \frac{\omega}{2} d \omega \sin \vartheta d \vartheta d \varphi, \quad \int_{G} d g=8 \pi^{2} .
$$

In the sense of this picture an "orientation distance"

$$
\tilde{\omega}=\tilde{\omega}\left(g_{1}, g_{2}\right)=\tilde{\omega}\left(g_{2}, g_{1}\right)
$$

exists between two points of the $G$-space given by the expression (Varshaloviĉ et al., 1975; Helming et al., 1987)

$$
\begin{aligned}
\cos \frac{\tilde{\omega}}{2}= & \cos \frac{\omega_{1}}{2} \cos \frac{\omega_{2}}{2}+\left(\vec{n}_{1} \cdot \vec{n}_{2}\right) \sin \frac{\omega_{1}}{2} \sin \frac{\omega_{2}}{2} \\
= & \cos \left(\frac{\alpha_{1}+\gamma_{1}}{2}-\frac{\alpha_{2}+\gamma_{2}}{2}\right) \cos \frac{\beta_{1}}{2} \cos \frac{\beta_{2}}{2} \\
& +\cos \left(\frac{\alpha_{1}-\gamma_{1}}{2}-\frac{\alpha_{2}-\gamma_{2}}{2}\right) \sin \frac{\beta_{1}}{2} \sin \frac{\beta_{2}}{2} .
\end{aligned}
$$

In practice the ODF $f(g)$ of a sample is determined for a finite set of $G$-space points (e.g. $5^{\circ}$-steps for $\left.\alpha, \beta, \gamma\right)$ :

$$
f\left(g_{m}\right), \quad g_{m} \in G, \quad m=1,2, \ldots, M .
$$

The large number of such ODF-data ( $M$ is a number of the order of many thousands) makes an obvious ODF interpretation very 


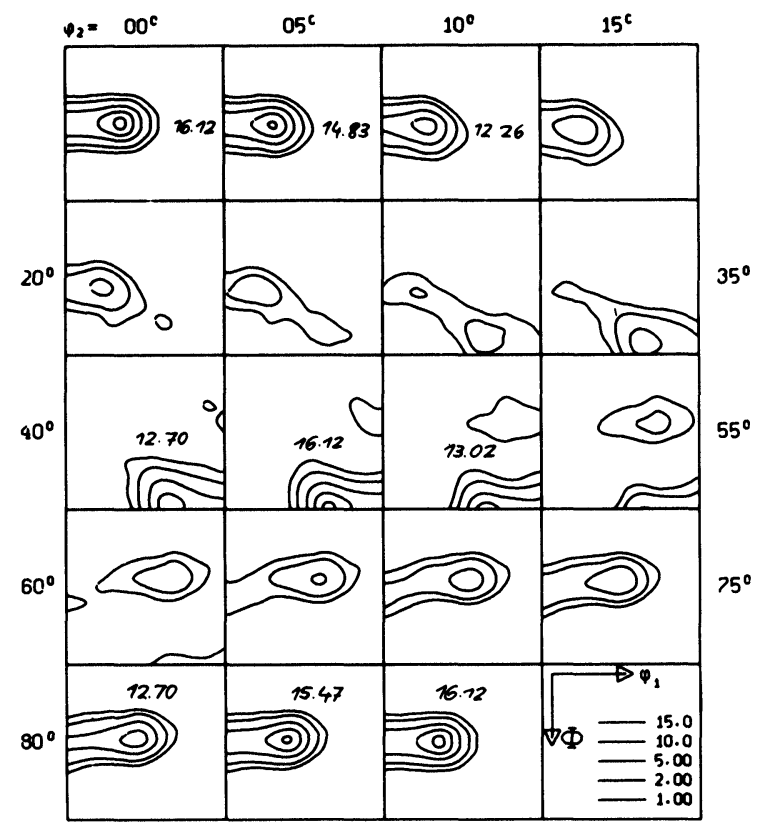

Figure 1 Orientation distribution $f(g)$ of a cold rolled copper $(9 \% \mathrm{Ge})$ alloy. $\varphi_{1} \triangleq \pi / 2-\alpha, \Phi \triangleq \beta, \varphi_{2} \triangleq \pi / 2-\gamma$. Pole figure data from RWTH Aachen. ODF reproduced and ghost corrected by the WIMV-method (cf. Wenk, 1985).

difficult. On the other hand (cf. Figure 1) regions of high magnitudes around a small number $K$ of preferred orientations $g_{k}^{0}$ $(k=1,2, \ldots, K)$ are characteristic for real distributions and it is desirable to interpret the ODF by such a small number of "texture components".

In order to realize this concept more quantitatively (concept of "data compression" or "component fit", cf. Lücke et al., 1986) the given ODF is approximatively represented by a set of $K$ model distributions $f\left(B_{k}, g_{k}^{0}, g\right)$ decreasing in a bell-like manner for orientations $g$ "far" from the centre of a component at $g_{k}^{0}$. The corresponding peak form is characterized by the symbol $B_{k}$ including a possible set of width-parameters.

The most simple model distribution can be constructed using so-called "central functions" $f(B, \tilde{\omega})$ depending on $g_{k}^{0}$ and $g$ by their orientation distance (5) $\tilde{\omega}=\tilde{\omega}\left(g_{k}^{0}, g\right)$ only. Using such a 
picture the ODF modelled is given by

$$
f(g)=F_{c}+\sum_{k=1}^{K} I_{k} f\left(B_{k}, \tilde{\omega}\left(g_{k}^{0}, g\right)\right)
$$

with $F_{c}$ a constant background ("phon", $0 \leqslant F_{c} \leqslant 1$ ), the component intensities $I_{k}\left(0 \leqslant I_{k} \leqslant 1\right)$, and

$$
\int_{G} f(g) d g=\int_{G} f\left(B_{k}, \tilde{\omega}\left(g_{k}^{0}, g\right)\right) d g=8 \pi^{2} ; \quad F_{c}+\sum_{k=1}^{K} I_{k}=1 .
$$

If for numerical calculations the harmonic method (cf. Bunge, 1982; Wenk, 1985; Matthies et al., 1987) is used:

$$
f(g)=\sum_{l=0}^{\infty} \sum_{m, n=-l}^{l} C_{l}^{m, n} D_{m, n}^{l}\left(g^{-1}\right),
$$

the corresponding Fourier coefficients $C_{l}^{m, n}$ are given by

with

$$
C_{l}^{m, n}=F_{c} \delta_{l, 0}+\sum_{k=1}^{K} I_{k} C_{l}^{m, n}(k),
$$

$$
\left.\begin{array}{l}
C_{l}^{m, n}(k)=C_{l}\left(B_{k}\right) D_{m, n}^{l^{*}}\left(g_{k}^{0-1}\right), \quad f\left(B_{k}, \tilde{\omega}\right)=\sum_{l=0}^{\infty} C_{l}\left(B_{k}\right) \mathscr{X}_{l}(\tilde{\omega}), \\
C_{l}\left(B_{k}\right)=\frac{2}{\pi} \int_{0}^{\pi} f\left(B_{k}, \tilde{\omega}\right) \mathscr{X}_{l}(\tilde{\omega}) \sin ^{2} \frac{\tilde{\omega}}{2} d \tilde{\omega}, \\
\mathscr{X}_{l}(\tilde{\omega})=\sin ((2 l+1) \tilde{\omega} / 2) / \sin (\tilde{\omega} / 2) .
\end{array}\right\}
$$

\section{MODEL DISTRIBUTIONS USED IN TEXTURE ANALYSIS}

The first model of the central type widely used in practice was suggested by Bunge ("Bunge's Gaussian distribution") and reads

$$
f(\Psi, \tilde{\omega})=N(\Psi) e^{-(\tilde{\omega} / \Psi)^{2}},
$$

with

$$
\begin{gathered}
N(\Psi) \approx 2 \sqrt{\pi} /\left[\Psi\left(1-e^{-(\Psi / 2)^{2}}\right)\right], \\
C_{l}(\Psi) \approx\left[e^{-(l \Psi / 2)^{2}}-e^{-((l+1) \Psi / 2)^{2}}\right] /\left(1-e^{-(\Psi / 2)^{2}}\right), \\
b=2 \sqrt{\ln 2} \Psi .
\end{gathered}
$$


In the paper $b$ always means the halfwidth (FWHM)-full width at half maximum) of the corresponding bell-shaped curve

$$
f(B, \tilde{\omega}=b / 2)=\frac{1}{2} f(B, \tilde{\omega}=0) .
$$

The symbol " $\approx "$ in (13) and (14) indicates that these approximations are valid for $b \ll \pi$ only.

There is another number of unpleasant properties of this distribution yet. So the analytically closed form (12) does not possess the property of symmetry of an orientation distance

$$
\tilde{\omega}=\pi+\varepsilon \triangleq \tilde{\omega}=\pi-\varepsilon .
$$

Moreover, due to the restriction $b \ll \pi$ the distribution cannot satisfactorily describe the whole width spectrum for bell-shaped curves beginning with very sharp peaks $(b \rightarrow 0)$ up to the random distribution $f(B, \tilde{\omega})=1$. But much more serious is the fact, that the most interesting related functions for texture analysis like pole figures cannot be found in an analytically closed form and have to be calculated by series of the type (9), (11) or (20). This leads to unpleasant problems of series termination errors and numerical accuracy (Matthies, 1988).

In order to overcome these difficulties the so called "standard functions" were suggested (Matthies, 1980). Two forms have been considered up to now. The "Gaussian standard function" is given by $\left(I_{l}(x)\right.$-modified Bessel functions):

$$
\begin{gathered}
f(S, \tilde{\omega})=N(S) e^{S \cos \tilde{\omega}}, \quad N(S)=1 /\left(I_{0}(S)-I_{1}(S)\right), \\
C_{l}(S)=N(S) \cdot\left[I_{l}(S)-I_{l+1}(S)\right], \\
0 \leqslant S<\infty ; \quad S=\ln 2 /\left[2 \sin ^{2}(b / 4)\right] \quad \text { for } \quad b \leqslant 2 \pi(\triangleq S \geqslant \ln \sqrt{2}) .
\end{gathered}
$$

The calculation of pole figures $(\vec{y}=(\vartheta, \varphi)$-sample direction, $\vec{h}_{i}=\left(\vartheta_{i}, \varphi_{i}\right)$-projection direction) for a given ODF leads to combinations of expressions of the type ("ideal pole figures")

$$
\mathscr{P}_{\overrightarrow{h_{i}}}(\vec{y})=\frac{1}{2 \pi} \int_{0}^{2 \pi} f\left(\left\{\varphi_{i}, \vartheta_{i}, \bar{\varphi}\right\}^{-1} \cdot\{\varphi, \vartheta, 0\}\right) d \bar{\varphi} .
$$

For a central function $f(B, \tilde{\omega})$ the corresponding series reads 
$\left(P_{l}(z)\right.$-Legendre polynomials):

$$
\mathscr{P}_{\vec{h}_{i}}(\vec{y})=P(B, z)=\sum_{l=0}^{\infty} C_{l}(B) P_{l}(z),
$$

with

$$
z=\vec{h}_{i} \cdot g^{0} \cdot \vec{y}
$$

For $f(S, \tilde{\omega})$ the $P(S, z)$ can be represented in a closed form:

$$
P(S, z)=e^{S(z-1) / 2} I_{0}(S(z+1) / 2) N(S),
$$

as was demanded for a "standard function".

The second type of such functions was called "Lorentzian standard function" (Matthies, 1982):

$$
f(t, \tilde{\omega})=\left(1-t^{2}\right) \frac{\left(1+t^{2}\right)^{2}+4 t^{2} \cos ^{2}(\tilde{\omega} / 2)}{\left[\left(1+t^{2}\right)^{2}-4 t^{2} \cos ^{2}(\tilde{\omega} / 2)\right]^{2}}, \quad 0 \leqslant t<1,
$$

with

$$
C_{l}(t)=(2 l+1) t^{2 l},
$$

and for $0<b \leqslant 2 \pi\left(\triangleq t^{*} \leqslant t<1\right)$

$c=\cos ^{2}(b / 4), \quad A=\left(19 c^{2}-34 c+19\right)^{1 / 2}$,

$t=\sqrt{\tau}-\sqrt{\tau-1}, \quad \tau^{*}=(5+\sqrt{17}) / 2$,

$\left.\left.\tau=\frac{2}{3} A \cos \left\{\frac{1}{3} \arccos \left[\left(-82 c^{3}+240 c^{2}-246 c+80\right) / A^{3}\right)\right]\right\}+\frac{5-4 c}{3}.\right\}$

The corresponding standard pole figure $P(t, z)$ reads (cf. (21))

$$
P(t, z)=\left(1-t^{4}\right) /\left(1-2 t^{2} z+t^{4}\right)^{3 / 2} \text {. }
$$

With the help of these standard functions many problems can be investigated in texture analysis. At this, especially their analytically closed form is to be underlined once more permitting among other things to realize numerical calculations with high accuracy. For details see Matthies et al., 1987.

Whereas the Gaussian standard function (16) is practically identical with Bunge's Gaussian distribution (12) for small halfwidths $b$, the Lorentzian standard distribution (23) differs from those. Normalized to equal magnitudes at $\tilde{\omega}=0$ the Lorentzian 


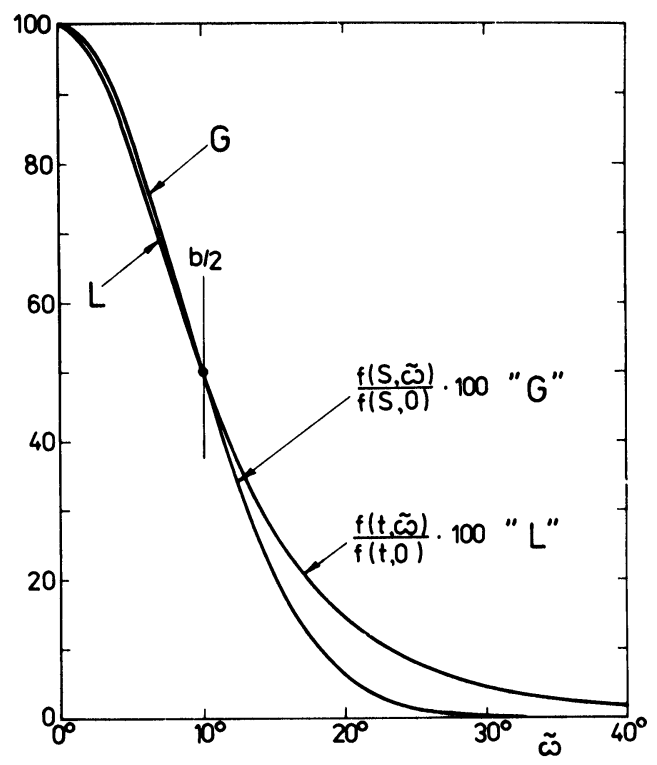

Figure 2 Lorentzian (23) and Gaussian (16) standard distributions with the same halfwidth $b=20^{\circ}(\triangleq S=45.625 ; t=0.87333)$ normalized to the altitude 100 .

curve will be more narrow than the Gaussian curves in the vicinity of the peak maximum $(\tilde{\omega}<b / 2)$. The Gaussian curves go extremely quickly to zero for $\tilde{\omega}>b / 2$, whereas the Lorentzian distribution possesses a long tail which disappears slowly. See Figure 2 . In this connection the phenomenon of form effects is of certain interest.

\section{FORM EFFECTS}

Describing a given distribution by a model curve, small deviations, especially at the flanks of a peak, seem to be not so aggravating from a first view. But the most important information on a texture component concerns its intensity, i.e. the integral about the peak region up to an orientation distance $\tilde{\omega}^{*}$ from the peak position at $g^{0}$ :

$$
I\left(\tilde{\omega}^{*}\right)=\int_{\tilde{\omega}\left(g^{0}, g\right) \leqslant \tilde{\omega}^{*}} f(g) d g /\left(8 \pi^{2}\right) .
$$


Table 1 The dependence of $I\left(\tilde{\omega}^{*}\right) \cdot 100 \%$ on $\tilde{\omega}^{*}$ for standard peaks with $b=$ $15^{\circ}$ (cf. (27))

\begin{tabular}{crr}
\hline$\tilde{\omega}^{*}$ & \multicolumn{1}{c}{$G$} & \multicolumn{1}{c}{$L$} \\
\hline $7.5^{\circ} \triangleq b / 2$ & $30 \%$ & $9 \%$ \\
$15.0^{\circ} \triangleq b$ & $87 \%$ & $32 \%$ \\
$22.5^{\circ} \triangleq 3 b / 2$ & $99 \%$ & $50 \%$ \\
$180^{\circ}$ & $100 \%$ & $100 \%$ \\
\hline
\end{tabular}

Due to the weighting factor $\sin ^{2} \tilde{\omega} / 2$ in (4) the flank regions of a peak give remarkable contributions to $I\left(\tilde{\omega}^{*}\right)$. Table 1 gives an impression how quickly a Gaussian $(G)$ or Lorentzian $(L)$ peak reaches its full intensity in the orientation space.

Now, if we have a Gaussian $f_{G}(\tilde{\omega})=M_{G} f(S, \tilde{\omega})$ and a Lorentzian peak $f_{L}(\tilde{\omega})=M_{L} f(t, \tilde{\omega})$ with the same halfwidth $b$ (e.g. $\left.b=15^{\circ}\right)$ and altitude the relations

$f_{G}(0)=f_{L}(0)=M_{G} f(S, 0)=M_{L} f(t, 0)$, i.e. $M_{L} / M_{G}=f(S, 0) / f(t, 0)$

follow. Although the two peaks shown in Figure 2 (such a picture is characteristic for ODF representations) do not differ very drastically the difference of the intensities $I\left(\tilde{\omega}^{*}\right)$ is more impressive. So we get, using the data from Table 1 and $f(S, 0) / f(t, 0)=3.45$ for $b=15^{\circ}: I_{L}\left(\tilde{\omega}^{*}=b / 2\right) / I_{G}\left(\tilde{\omega}^{*}=b / 2\right)=0.99, I_{L}(b) / I_{G}(b)=1.25$ and $I_{L}(3 b / 2) / I_{G}(3 b / 2)=1.74$. I.e. even interpreting a texture component in a finite region around its maximum only, remarkable errors may appear in the evaluation of its intensity if the model distribution does not well describe the flank regions (Matthies, 1982).

Of course, in fit procedures it is much more pleasing to work with simple Gaussian model peaks, and at the end every complicated distribution can well be described by a set of peaks of a form once fixed as it was underlined by Lücke et al., 1986. But the investigation of the true form of texture components is not an academic question only, although the present quality of the experimental data in regions of small magnitudes complicates the analysis of such details. Just the form of texture components may contain valuable informations on mechanisms of texture development, and it seems to be worth to underline that the in principle available information on the form of peaks is not used in texture analysis up to now. 
Unlike this situation in other fields of physics (cf. Egelstaff, 1965) the analysis of peak forms is often the only possibility to get valuable information on the mechanisms of interest.

\section{THE TERM “NORMAL DISTRIBUTION”}

In connection with the discussion of peak forms and model distributions recently a new interesting aspect appears. Speaking about Gaussian distributions it is not without interest to ask what the term "Gaussian" generally means. Indeed, Bunges's Gaussian distribution (12) is only an analogon of the one-dimensional "normal distribution"

$$
f_{N}(x)=\frac{1}{\sqrt{2 \pi d}} e^{-(x-\bar{x})^{2} / 2 d}
$$

investigated by Gauss and widely used in statistics. The Gaussian standard distribution (16) was simply named so due to its analogical behaviour for small halfwidths. But of own interest is the question what a form the normal distribution (i.e. the "truly" Gaussian distribution) possesses in the three-dimensional $G$-space with its nontrivial metric (2) or (4). This is so more of importance because the normal distribution in a given space plays a special role due to its connection with the so-called central limit theorem of probability theory.

As it will be explained in more detail below this theorem considers the form of the probability distribution of a quantity describing a sum of a large number of independent quantities all possessing their own probability distributions. Now, understanding a peak in an ODF as a distribution describing a result of a sum of steps of texture development mechanisms applied to a large number of crystallites in the sample it becomes evident why the normal distribution in connection with peak forms is of direct interest for texture analysis.

Valuable investigations dealing with the form of the normal distribution in the $G$-space were recently performed by T. I. Savjolova, 1985. Using the apparatus of rather abstract mathematics this distribution was exactly derived by her for the common 
( $g=\{\alpha, \beta, \gamma\})$ three-dimensional case. However the final results could be represented in form of Fourier series only.

Because we are mainly interested in practical numerical applications, in analytically closed expressions, and (if possible) in simplified derivations of the corresponding relationships, additional investigations were performed by us concerning the normal distribution. At this, the case of central distributions $f\left(B, \tilde{\omega}\left(g^{0}, g\right)\right)$ will only be considered below that leads to a one-dimensional problem.

\section{THE CENTRAL LIMIT THEOREM}

For our purposes we formulate this theorem (cf. Richter, 1956) in the following simplified form. Be $x_{1}, x_{2}, \ldots, x_{q}, \ldots, x_{n}$ a set of independent, accidental quantities characterized by their probability distributions

with

$$
\varphi_{1}\left(x_{1}\right), \varphi_{2}\left(x_{2}\right), \ldots, \varphi_{q}\left(x_{q}\right), \ldots, \varphi_{n}\left(x_{n}\right),
$$

$$
\bar{x}_{q}=\int_{-\infty}^{\infty} x \varphi_{q}(x) d x, \quad d_{q}=\int_{-\infty}^{\infty}\left(x-\bar{x}_{q}\right)^{2} \varphi_{q}(x) d x,
$$

then the probability distribution $\Phi_{n}(X)$ of the quantity

$$
\begin{array}{r}
X=X_{n}=\sum_{q=1}^{n} x_{q}, \text { with } \bar{X}_{n}=\int_{-\infty}^{\infty} X \Phi_{n}(X) d X, \\
D_{n}=\int_{-\infty}^{\infty}\left(X-\bar{X}_{n}\right)^{2} \Phi_{n}(X) d X,
\end{array}
$$

goes for $n \gg 1$ and any distributions $\varphi_{q}\left(x_{q}\right)$ (belonging to a class of functions obeying relatively weak conditions) to the normal distribution $F(X)$ of the form (28)

with

$$
F(X)=\frac{1}{\sqrt{2 \pi D}} e^{-(X-\bar{X})^{2} / 2 D} \approx \Phi_{n}(X) \quad(n \gg 1),
$$

$$
\bar{X}=\bar{X}_{n}=\sum_{q=1}^{n} \bar{x}_{q}, \quad \text { and } \quad D=D_{n}=\sum_{q=1}^{n} d_{q} .
$$


The exact expression for $\Phi_{n}(X)$ :

$$
\begin{aligned}
\int_{-\infty}^{\infty} d X_{n-1} & \int_{-\infty}^{\infty} d X_{n-2} \cdots \\
& \int_{-\infty}^{\infty} d X_{1} \varphi_{n}\left(X-X_{n-1}\right) \cdots \varphi_{q}\left(X_{q}-X_{q-1}\right) \cdots \varphi_{1}\left(X_{1}\right)
\end{aligned}
$$

follows successively from the simplest case $(n=2)$

$$
\Phi_{2}(X)=\int_{-\infty}^{\infty} \varphi_{2}\left(X-X_{1}\right) \varphi_{1}\left(X_{1}\right) d X_{1} ; \quad X_{1}=x_{1} .
$$

Now, considering a set of identical distributions $\varphi_{q}^{(n)}(x)=\varphi^{(n)}(x)$, with

$$
\bar{x}_{q}^{(n)}=0, \quad \text { and } \quad d_{q}^{(n)}=\dot{d}^{(n)}=d_{0} / n,
$$

we will get

$$
\Phi_{n}(X) \approx F_{0}(X)=\frac{1}{\sqrt{2 \pi d_{0}}} e^{-X^{2} / 2 d_{0}}, \quad n \gg 1
$$

an expression preserving the form of the normal distribution (31), but with a finite dispersion $D=d_{0}$ for $n \rightarrow \infty$ also. An example demonstrating how quickly the normal distribution arises is given in Figure 3. Using this trick we can consequently find the form of the considered normal distribution in more complicated spaces, too, if we are able to determine the limit $n \rightarrow \infty$ of convolution integrals of type (33) in these spaces.

In the simplest way this can be realized using the Fourier representation given for the common one-dimensional case by the expressions

$$
\varphi(x)=\int_{-\infty}^{\infty} h(k) e^{i 2 \pi k x} d k, \quad h(k)=\int_{-\infty}^{\infty} \varphi(x) e^{-i 2 \pi k x} d x,
$$

with the valuable property for convolution integrals of type (33)

$$
\Phi_{n}(X)=\int_{-\infty}^{\infty} H_{n}(k) e^{i 2 \pi k X} d k, \quad H_{n}(k)=\prod_{q=1}^{n} h_{q}(k) .
$$




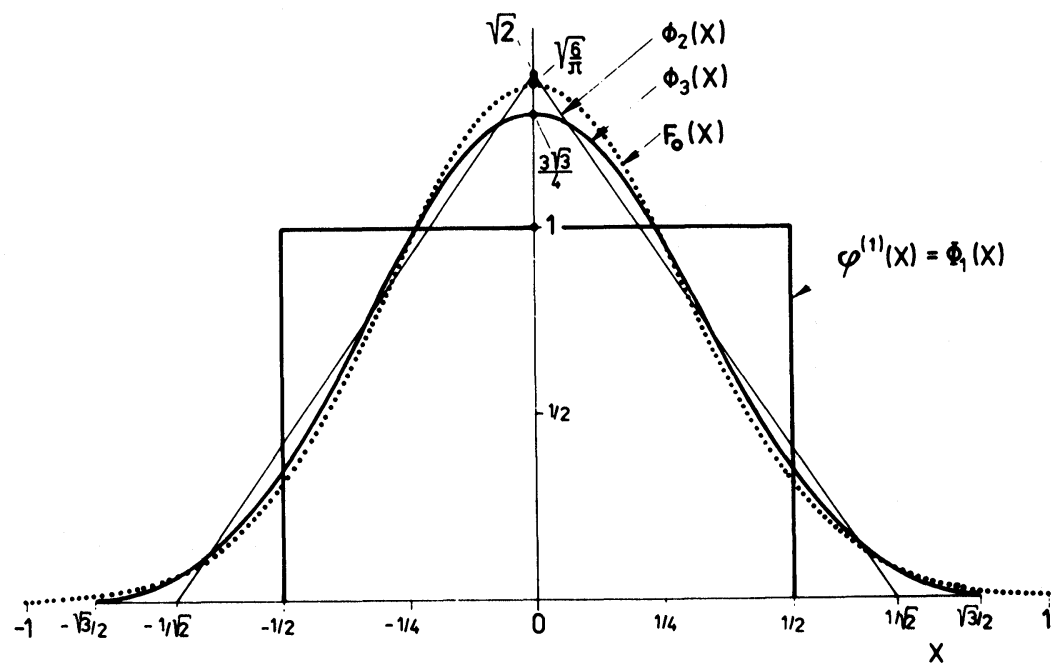

Figure 3 Demonstration of the convergence of $\Phi_{n}(X)$ (33) to the normal distribution $F_{0}(x)$ (36). For $\varphi^{(n)}(x)$, rectangular distributions with $d^{(n)}=1 /(12 n)$ (cf. (35)) are chosen. $\Phi_{n}(X)(n=1,2,3)$ are shown. For 10 convolutions, $\Phi_{n}(X)$ is practically identical with $F_{0}(x)$.

\section{CONVOLUTION INTEGRALS IN THE G-SPACE}

In difference to the Euclidian one-dimensional case described above, orientations $g_{q}$ (a set of rotations) are to be considered now instead of the $x_{q}$. A "sum" of $g_{1}$ and $g_{2}$ is to be understood as $G_{2}=g_{2} \cdot g_{1}$. At this, additional attention is necessary due to $g_{2} \cdot g_{1} \neq g_{1} \cdot g_{2}$ as a rule. A "difference" $g_{2}-g_{1}$ we define as $g_{2} \cdot g_{1}^{-1}$. With these presumptions a convolution integral of type (34) is given by

$$
\Phi_{2}\left(G_{2}\right)=\int_{G} \varphi_{2}\left(G_{2} \cdot g_{1}^{-1}\right) \varphi_{1}\left(g_{1}\right) d g_{1} /\left(8 \pi^{2}\right) .
$$

Now, using the series representation (9) we get

$$
\begin{aligned}
& \sum_{l, m, n} C_{l}^{m, n}\left(\Phi_{2}\right) D_{m, n}^{l}\left(G_{2}^{-1}\right)=\int_{G} \sum_{l, m, n} C_{l}^{m, n}\left(\varphi_{2}\right) D_{m, n}^{l}\left(g_{1} \cdot G_{2}^{-1}\right) \\
& \times \sum_{l^{\prime}, m^{\prime}, n^{\prime}} C_{l^{\prime}}^{m^{\prime}, n^{\prime}}\left(\varphi_{1}\right) D_{m^{\prime}, n^{\prime}}^{l^{\prime}}\left(g_{1}^{-1}\right) d g_{1} /\left(8 \pi^{2}\right)
\end{aligned}
$$


leading to

$$
C_{l}^{m, n}\left(\Phi_{2}\right)=\sum_{n^{\prime}} C_{l}^{m, n^{\prime}}\left(\varphi_{2}\right) C_{l}^{n^{\prime}, n}\left(\varphi_{1}\right) /(2 l+1)
$$

At the end, for central functions $f(B, \tilde{\omega})$ depending on the orientation distance $\tilde{\omega}\left(g_{q}^{0}, g\right)$, only, with $g_{q}^{0}=\{0,0,0\}$ (cf. $\bar{x}_{q}=0$ in (35)) and using $D_{m, n}^{l}(\{0,0,0\})=\delta_{m, n}$ as well as (11) the asked result reads:

$$
C_{l}\left(\Phi_{2}\right)=(2 l+1)\left[C_{l}\left(B_{1}\right) /(2 l+1)\right]\left[C_{l}\left(B_{2}\right) /(2 l+1)\right] .
$$

Comparing this formula with the relationship (38) for $n=2$ ( $C \triangleq$ $H, h ; l \triangleq k)$ a full analogy to the previously considered onedimensional case is seen. Therefore in order to find the form of the normal distribution in the $G$-space we can use the program explained in context with (36).

\section{THE NORMAL DISTRIBUTION IN THE G-SPACE (CENTRAL FUNCTIONS)}

The problem is to calculate for $n \rightarrow \infty$ the multiple convolution intergral of type (33) using, as for (36), identical $\varphi_{q}^{(n)}(\tilde{\omega})=\varphi^{(n)}(\tilde{\omega})$ with decreasing dispersion (or peak width) for increasing $n$. Taking for $\varphi^{(n)}(\tilde{\omega})$ the simplest bell-shaped distribution (12) we get using (14) and $\Psi^{(n)}=\Psi_{0} / \sqrt{n}$

$$
C_{l}\left(\Psi^{(n)}\right) \approx(2 l+1)\left[1-l(l+1)\left(\Psi_{0} / 2\right)^{2} \cdot \frac{1}{n}\right], \quad n \gg 1 .
$$

For $n$ convolutions

$$
C_{l}\left(\Phi_{n}\right)=(2 l+1) \prod_{q=1}^{n}\left[C_{l}\left(B_{q}\right) /(2 l+1)\right]
$$

follows from (42). Finally, with the help of (43), we get for the $C_{l}$ coefficients of the considered normal distribution 


$$
\begin{aligned}
& \left(\kappa=l(l+1)\left(\Psi_{0} / 2\right)^{2} \equiv l(l+1) \varepsilon\right) \text { the result } \\
& \qquad \begin{aligned}
C_{l}(\text { normal }) & =\lim n \rightarrow \infty(2 l+1) \prod_{q=1}^{n}\left[1-\frac{\kappa}{n}\right] \\
& =\lim n \rightarrow \infty(2 l+1)\left[1-\frac{\kappa}{n}\right]^{n} \\
& =(2 l+1) e^{-\varepsilon l(l+1)}=C_{l}(\varepsilon),
\end{aligned}
\end{aligned}
$$

first given by Savjolova. Introducing for this distribution the notation $f(\varepsilon, \tilde{\omega})$ we have according to (11)

$$
f(\varepsilon, \tilde{\omega})=\sum_{l=0}^{\infty} C_{l}(\varepsilon) \mathscr{X}_{l}(\tilde{\omega}) .
$$

Of course, due to the central limit theorem, the result (45) does not depend on the fact that we have used (43)-an expression following from Bunge's Gaussian distribution (cf. (14)). The same result will arise if e.g. for $\varphi^{(n)}(\tilde{\omega})$ the standard Gaussian distribution $(16,17)$ is chosen, with $S^{(n)}=S_{0} \cdot n, \varepsilon=1 /\left(2 S_{0}\right)$.

The characteristic property of the normal distribution $f(\varepsilon, \tilde{\omega})$ is the extremely simple form of its $C_{l}$ coefficients (45). Moreover, as for the standard Gaussian or Lorentzian distributions, the whole width-spectrum of a bell-shaped curves can be described by the width parameter $\varepsilon$. For $\varepsilon \rightarrow 0$ we get a delta function and $\varepsilon \rightarrow \infty$ describes the case of the random distribution. Unfortunately the explicit form of $f(\varepsilon, \tilde{\omega})$ cannot be seen from the series (46) in contradiction to the analytically closed expressions for the model distributions (12), (16), and (23) considered up to now. Especially unfavourable is the fact that an explicit relationship is missing between the width parameter $\varepsilon$ and the halfwidth $b$ of the corresponding bell-shaped curve (cf. (15), (18), (25)).

In order to overcome this disadvantage the series (46) was carefully analysed. The following approximative expressions were found to describe $f(\varepsilon, \tilde{\omega})$ with very high accuracy for $\varepsilon<1(\underline{\underline{\Lambda}} b<$ $\left.210^{\circ}\right)$ :

$$
\begin{gathered}
f(\varepsilon, \tilde{\omega}) \approx \sqrt{\pi} \varepsilon^{-3 / 2} e^{\varepsilon / 4} e^{-(\tilde{\omega} / 2)^{2} / \varepsilon} \\
\times\left[\tilde{\omega}-e^{-\pi^{2} / \varepsilon}\left\{(\tilde{\omega}-2 \pi) e^{\pi \tilde{\omega} / \varepsilon}+(\tilde{\omega}+2 \pi) e^{-\pi \tilde{\omega} / \varepsilon}\right\}\right] /\left(2 \sin \frac{\tilde{\omega}}{2}\right), \\
\varepsilon \approx b^{2} /\{16 \ln [b /(2 \sin (b / 4))]\} .
\end{gathered}
$$


The region $b<210^{\circ}$ is sufficient for all practical problems connected with bell-shaped distributions. For very flat curves $(\varepsilon>1)$ the series (46) converges rapidly due to the exponential factor $l(l+1)$ in (45). The derivation of the formula (47) is given in the appendix A.

In Figure 4 the three Gaussian distributions discussed are shown for $b=60^{\circ}$. As can be seen even for this relatively large halfwidth the normal distribution is practically identical with Bunge's Gaussian (differences lower than $0.01 \%$ ). For lower halfwidths typical in practice, the deviations are even much smaller. Therefore, working with the harmonic apparatus for fit problems as in (Lücke et al., 1986), it can be recommended to use for Bunge's Gaussian distribution the simpler and (especially for large $l$ ) more exact Fourier coefficients (45) together with the relationships (15) and (48). The standard Gaussian distribution (16) differs from the first two distributions for large halfwidths, only. Already for $b=20^{\circ}$ the difference of the maximum values decreases to $1 \%$ and will be much smaller for lower $b$.

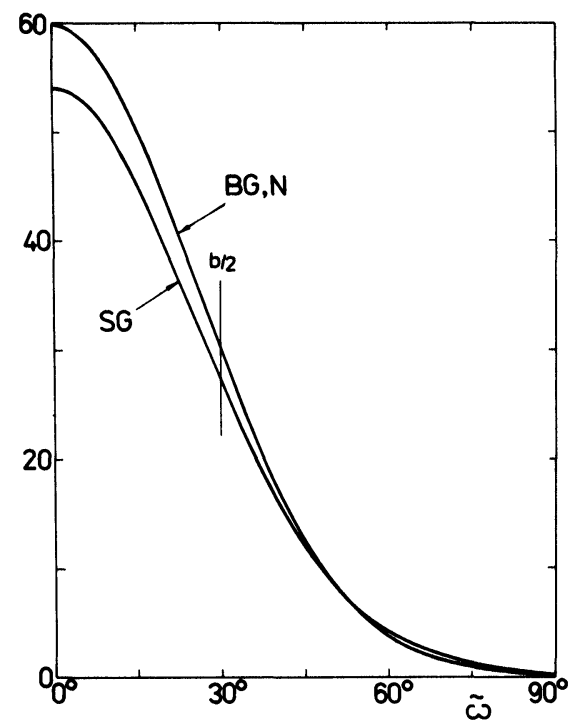

Figure 4 Comparison of the three "Gaussian" distributions in the $G$-space for a relatively large halfwidth $b=60^{\circ}\left(\triangleq \Psi=36.03^{\circ} ; S=5.174 ; \varepsilon=0.09727\right)$. "SG"Gaussian standard distribution (16). Bunge's Gaussian distribution ("BG" (12)) and the normal distribution ("N" (47)) can optically be distinguished for larger halfwidths, only. For the given example, we have $f(\Psi, 0)=59.87 ; f(\varepsilon, 0)=59.86$. 
As was already explained, the advantage of the standard distributions (16) and (23) is the knowledge of analytically closed expressions for the most important related functions in texture analysis. In spite of the valuable approximation for the normal distribution (47), the calculation of its pole figures is still restricted to series (20) like those for Bunge's Gaussian distribution, and is therefore affected with the already mentioned numerical problems.

\section{SPECIFIC PROPERTIES OF THE LORENTZIAN DISTRIBUTION}

As can directly be shown using (37) and (38) the convolution of two common normal distributions (28) with $\bar{x}_{1}=\bar{x}_{2}=0$ exactly leads to a normal distribution again, with the resulting dispersion $D=d_{1}+$ $d_{2}$ and $\bar{X}=0$. An analogous property we will get for the normal distribution $f(\varepsilon, \tilde{\omega})$ in the $G$-space. Indeed, from (45) and (42) it follows $\varepsilon=\varepsilon_{1}+\varepsilon_{2}$.

The name "Lorentzian" standard distribution was first chosen for (23) by purely qualitative reasons. As it was already discussed in connection with Figure 2 the Lorentzian standard distribution is more narrow in the vicinity of the top of its peak than a Gaussian distribution with the same halfwidth $b$ and altitude. Moreover, it possesses a long tail which decreases only slowly with increasing distances from the position of the peak maximum. The classic Gaussian (28) and Lorentzian curves $(\bar{x}=0)$

$$
f_{L}(x)=\frac{2}{b \pi\left[1+(2 x / b)^{2}\right]}
$$

show the same properties. However, the analogy of the two Lorentzian curves (23) and (49) is much closer yet. Indeed, considering the convolution of two Lorentzian distributions (49) using (37) and (38) it exactly follows that the resulting curve will also be a Lorentzian curve with the halfwidth $b=b_{1}+b_{2}$. The same we get for the Lorentzian (23) in the $G$-space using the equations (42) and (24) leading to $t=t_{1} \cdot t_{2}$. From (25) the approximation $t \approx \exp (-b / \sqrt{6})$ can be found for small $b$. So, in full analogy to the classic Lorentzian curve, it follows $b \approx b_{1}+b_{2}$.

From a first sight there seems to be a contradiction to the central 
limit theorem considered in paragraph 5. Indeed, using for the $\varphi_{q}(x)$ in (33) the Lorentzian curve (49) we will never get the normal distribution for large $n$ because the convolution of Lorentzian distributions leads to a Lorentzian curve again. The same is valid for the standard Lorentzian distribution (23). However, in connection with the formulation of the central limit theorem it was remarked that the functions $\varphi_{q}(x)$ have to obey some conditions. The Lorentzian curve is right an example not obeying these conditions (cf. appendix B).

The last circumstance is not of purely mathematical interest, only. As it was already discussed in paragraph 3, the form of texture components may contain certain informations on the mechanisms of texture development. Because the Lorentzian distribution is (like the normal distribution) invariant in regard of convolutions connected with "sums" of reorientations, it is not to exclude that texture components with a Lorentzian peak form may arise for certain mechanisms. Due to the continuous improvement of statistics in numerical calculations modelling processes of texture development, a direct analysis of the connection of peak forms and mechanisms of texture development becomes a real and interesting problem, now.

\section{Acknowledgement}

A substantial part of the reported investigations was realized during the yearly working stays of S. M. at the University of Metz. The authors wish to thank Prof. C. Esling for his permanent interest and support.

\section{APPENDIX A}

The series for the normal distribution $(45,46)$

$$
\begin{aligned}
f(\varepsilon, \omega) & =\left[\sum_{l=0}^{\infty}(2 l+1) e^{-\varepsilon l(l+1)} \sin \left((2 l+1) \frac{\omega}{2}\right)\right] / \sin \frac{\omega}{2} \\
& \equiv Z(\varepsilon, \omega) / \sin \frac{\omega}{2} .
\end{aligned}
$$

converges relatively slowly for small halfwidths $b \ll \pi(\triangleq \varepsilon \ll 1)$. For 
this reason we tried to find another series converging more rapidly in this case.

At first we can write

$$
Z(\varepsilon, \omega)=-2 \frac{\delta}{\delta \omega} \sum_{l=0}^{\infty} e^{-\varepsilon l(l+1)} \cos \left((2 l+1) \frac{\omega}{2}\right)
$$

Introducing the new variables

$$
v=\frac{\omega}{2 \pi}, \quad q=e^{-\varepsilon}
$$

we get

$$
Z(\varepsilon, \omega)=-\frac{1}{\pi} \frac{\delta}{\delta v} \sum_{l=0}^{\infty} q^{l(l+1)} \cos ((2 l+1) \pi v) .
$$

Comparing this expression with the series of Jacobi's $\theta_{2}$ function (Erdélyi, 1953)

$$
\theta_{2}(v, q)=2 q^{1 / 4} \sum_{n=0}^{\infty} q^{n(n+1)} \cos ((2 n+1) \pi v)
$$

it follows

$$
Z(\varepsilon, \infty)=-\frac{q^{-1 / 4}}{2 \pi} \frac{\delta}{\delta v} \theta_{2}(v, q)
$$

Although this allows to express the series (A1) by a known special function, nothing is earned as long as $\theta_{2}$ can only be calculated, by the series (A5), possessing the same bad convergence for $\varepsilon \ll 1$ $(\triangleq q \approx 1)$ as the initial form (A1). Fortunately, there is a special transformation leading to series of type (A5) with $\bar{q} \ll 1$ for $\varepsilon<1$. With

$$
\tau=\frac{-i}{\pi} \ln q, \quad \bar{v}=v / \tau, \quad \bar{\tau}=\frac{-1}{\tau},
$$

and

$$
\bar{q}=e^{-i \pi / \tau}=e^{\pi^{2} / \ln q}=e^{-\pi^{2} / \varepsilon}
$$

the following equation is valid:

$$
\begin{aligned}
\theta_{2}(v, q) & =\theta_{2}(v \mid \tau)=\theta_{4}(\bar{v} \mid \bar{\tau}) e^{i \pi \bar{v}^{2} / \bar{\tau}} \sqrt{\bar{\tau} / i} \\
& =\theta_{4}(\bar{v}, \bar{q}) e^{-i \pi v^{2} / \tau} \sqrt{i / \tau}
\end{aligned}
$$


I.e. we have

$$
\frac{\delta}{\delta v} \theta_{2}(v, q)=\sqrt{i} \tau^{-3 / 2} e^{-i \pi v^{2 / \tau}}\left[\frac{\delta}{\delta \bar{v}} \theta_{4}(\bar{v}, \bar{q})-i 2 \pi v \theta_{4}(\bar{v}, \bar{q})\right] .
$$

At the end, using the series

$$
\theta_{4}(\bar{v}, \bar{q})=1+\sum_{n=1}^{\infty}(-1)^{n} \bar{q}^{n^{2}} \cos (2 n \pi \bar{v})
$$

we get by $(\mathrm{A} 10,6,3)$ the asked result

$$
\begin{aligned}
& Z(\varepsilon, \omega)=\frac{\sqrt{\pi}}{2} e^{\left(\varepsilon-\omega^{2} / \varepsilon\right) / 4} \varepsilon^{-3 / 2} \\
& \quad \times\left\{\omega+\sum_{n=1}^{\infty}(-1)^{n} e^{-\pi^{2} n^{2} / \varepsilon}\left[(\omega-2 \pi n) e^{n \pi \omega / \varepsilon}+(\omega+2 \pi n) e^{-n \pi \omega / \varepsilon}\right]\right\}
\end{aligned}
$$

providing the approximation (47) for $f(\varepsilon, \tilde{\omega})$.

Except $\omega \approx \pi$ (where $Z(\varepsilon, \omega)$ itself is small due to the factor $\left.\exp \left(-\omega^{2} / 4 \varepsilon\right)\right)$ the sum in the last expression can usually be neglected in comparison with $\omega$. Indeed, already for a relatively large halfwidth $b=60^{\circ}(\wedge \varepsilon \approx 0.1)$ the factor $\exp \left(-\pi^{2} n^{2} / \varepsilon\right)$ is of the order of $10^{-43 n^{2}}$, only. For $b=15^{\circ}(\wedge \varepsilon \approx 0.006)$ we get $10^{-695} n^{2}$ correspondingly.

\section{APPENDIX B}

It is rather trivial why the central limit theorem does not work in the case of the common Lorentzian curve (49). In connection with (29), we have of course supposed that the dispersions $d_{q}$ exist. But (49) provides an infinite value due to the infinite integration boundaries in (29) and the long tail of the Lorentzian distribution disappearing too slowly.

This difficulty does not exist for the finite $G$-space (1), (3). For small halfwidths $(b \ll \pi$, i.e. $t \approx 1)$

$$
\begin{aligned}
d=\int_{G} \tilde{\omega}^{2} f(t, \tilde{\omega}) d g /\left(8 \pi^{2}\right)=\frac{2}{\pi} \int_{0}^{\pi} \tilde{\omega}^{2} f(t, \tilde{\omega}) \sin ^{2} \frac{\tilde{\omega}}{2} d \tilde{\omega} \\
\\
\approx\left(8 \ln 2-\pi^{2} / 6\right)\left(1-t^{2}\right)
\end{aligned}
$$


can be found for the standard Lorentzian distribution (23). Therefore, using

$$
t^{(n)}=1-\frac{1}{n} d_{0} /\left(16 \ln 2-\pi^{2} / 3\right)=1-2 \frac{\delta}{n}
$$

our working condition

$$
D=\lim n \rightarrow \infty D_{n}=\lim n \rightarrow \infty \sum_{q=1}^{n} d_{q}^{(n)}=\lim n \rightarrow \infty n d_{0} / n=d_{0}
$$

used in connection with $(35,36)$, is obeyed. But this is not sufficient in order to get the normal distribution for a sort of $\varphi^{(n)}(x)$. At first, $\varphi^{(n)}(x)$ has to go to a delta function for $n \rightarrow \infty$. This condition is also obeyed for $f(t, \tilde{\omega})$ with $t^{(n)}$ from (B2). The remaining demand concerns the velocity characterizing how quickly the tail of $\varphi^{(n)}(x)$ decreases with increasing $n$. In a simplified formulation, sufficient for our purposes, we can say that $n \cdot \varphi^{(n)}(\tilde{\omega}=\pi)$ has to disappear for $n \rightarrow \infty$. Just this condition is not obeyed for $f(t, \tilde{\omega})(23)$ due to

$$
\lim n \rightarrow \infty n \cdot f\left(t^{(n)}, \pi\right)=\lim n \rightarrow \infty n\left(1-t^{(n) 2}\right) /\left(1+t^{(n) 2}\right)^{2}=\delta \neq 0 .
$$

\section{References}

Bunge, H. J. (1982). Texture Analysis in Materials Science. Butterworths, London.

Egelstaff, P. A., Ed. (1965). Thermal Neutron Scattering. Academic Press, New York.

Erdélyi, A., Ed. (1953). Higher Transcendental Functions (Vol. II). McGraw-Hill Book Company, New York.

Helming, K., Matthies, S. and Vinel, G. W. (1987). Proc. of the 8th ICOTOM. AIME, in press.

Lücke, K., Pospiech, J., Jura, J. and Hirsch, J. (1986). Z. Metallkde. 77, 312-321.

Matthies, S. (1980). Phys. Stat. Sol. (b) 101, K111-K115.

Matthies, S. (1982). Phys. Stat. Sol. (b) 112, 705-716.

Matthies, S., Vinel, G. W. and Helming, K. (1987). Standard Distributions in Texture Analysis. Akademieverlag, Berlin.

Matthies, S. (1988). Textures and Microstructures, 8-9, 115-129.

Richter, H. (1956). Wahrscheinlichkeitstheorie. Springer Verlag, Berlin.

Savjolova, T. I. (1985). Preface to Novye Metody Issledovanija Tekstury Polikristalliceskich Materialov. Metallurgija, Moscow.

Varshalović, D. A., Moskalev, A. N. and Chersonskij, V. K. (1975). Kvantovaja Teorija Uglovogo Momenta. Izd. Nauka, Leningrad.

Wenk, H. R., Ed. (1985). Preferred Orientation in Deformed Metals and Rocks: An Introduction to Modern Texture Analysis. Academic Press, San Diego. 